Canadian Journal of Higher Education Revue canadienne d'enseignement supérieur

Volume 46, No. 3, 2016, pages 18 - 40

\title{
Analyse typologique des plans stratégiques des universités québécoises
}

Catherine Larouche

Université du Québec à Chicoutimi

Denis Savard

Université Laval

Lucie Héon

Université Laval

Jean-Joseph Moisset

Université Laval

\section{Résumé}

La présente recherche consiste à vérifier l'applicabilité de la typologie de Larouche et ses collaborateurs à des activités de planification stratégique des établissements d'enseignement supérieur. Cette typologie, développée en vue de l'évaluation de la performance, comporte sept types de conceptions définies en fonction de sept dimensions. Une analyse documentaire de 17 plans stratégiques, menée selon des critères de clarté et consistance logique, d'exhaustivité et d'utilité montre que cette typologie peut s'avérer éclairante lors des processus de planification : les conceptions sont définies assez clairement pour être repérables dans les plans stratégiques, elles recouvrent des réalités signifiantes présentes dans les documents analysés et la typologie permet d'appréhender des contextes complexes (alignement stratégique) et s'avère assez sensible pour rendre compte de la diversité des situations (caractérisation des établissements). 


\begin{abstract}
This research tests the applicability of the typology proposed by Larouche and his collaborators in higher education strategic planning. The typology developed for the assessment of performance, features seven types of concepts defined in terms of seven dimensions. A literature review of 17 strategic plans, conducted according to criteria of clarity and logical consistency, completeness, and usefulness shows that the typology may prove enlightening in the planning process. The results demonstrate that the conceptions are defined clearly enough to be detected in the strategic plans, and these conceptions cover meaningful realities in the documents analyzed, and the typology allows us to understand complex contexts (strategic alignment) that it is sensitive enough to reflect a diversity of situations (characterization of institutions).
\end{abstract}

\title{
Introduction
}

La typologie des conceptions des universités de Larouche et ses collaborateurs (Larouche, 2011; Larouche, Savard et Héon, 2014; Larouche, Savard, Héon et Moisset, 2012; 2014) a été développée en vue d'évaluer la performance des établissements universitaires dans le respect de leur complexité et de leur spécificité. La validation dont elle a fait l'objet a révélé que non seulement elle s'avère pertinente à l'occasion d'actions d'évaluation, mais qu'elle offre aussi un potentiel intéressant pour les processus de planification et d'analyse institutionnelles : les diverses conceptions fondant la typologie permettraient de situer les enjeux et d'identifier des objectifs en phase avec la mission, la vision et les valeurs des établissements et clarifieraient aussi les bases à partir desquelles les processus de planification se déploient.

Cet article vise à vérifier de manière empirique l'applicabilité de cette typologie aux exercices de planification, par le biais d'une analyse de contenu des plans stratégiques des universités québécoises. Nous utilisons pour en mesurer la pertinence les trois critères de validation d'une typologie proposés par Sauvé (1992) : clarté et consistance logique, exhaustivité et utilité. Plus spécifiquement, nous cherchons à répondre aux questions suivantes :

1. Clarté et consistance logique. Les conceptions de la typologie sont-elles assez clairement définies et possèdent-elles une consistance logique suffisante pour permettre de classer de façon univoque les différents éléments retrouvés dans les plans stratégiques?

2. Exhaustivité. La typologie permet-elle de couvrir l'ensemble des éléments présents dans les plans stratégiques? L'ensemble des conceptions de la typologie s'y retrouvent-elles? Quelles sont les caractéristiques des éléments ne pouvant être associés à une conception spécifique?

3. Utilité. La typologie possède-t-elle une sensibilité suffisante pour permettre le diagnostic et détecter la variabilité dans les différentes situations de planification, et donc pour constituer un outil d'analyse utile?

Après avoir présenté la problématique et décrit la typologie utilisée des conceptions des universités, nous définissons les concepts de mission, vision et valeurs (éléments analysés dans cet article) dans le cadre plus général de la gestion axée sur les résultats 
et de la planification stratégique. Nous explicitons ensuite le cadre d'analyse spécifique à notre étude, soit la définition des critères de validité d'une typologie et la présentation de la méthodologie employée (une analyse documentaire). Nous présentons et discutons dans la dernière partie les résultats de l'analyse des plans stratégiques (mission, vision, valeurs) en fonction de la typologie et des critères de validité d'une typologie.

\section{Problématique}

Les universités sont incitées à respecter, à l'instar des organismes publics, les principes d'une « saine gestion » et à adopter des pratiques inspirées de la gestion axée sur les résultats. Au Québec, la Loi sur les établissements d'enseignement de niveau supérieur $^{1}$ adoptée en 1995 et la Loi sur l'administration publique ${ }^{2}$ adoptée en 2000 imposent aux organismes l'adoption d'un plan stratégique, obligatoirement public, et le dépôt d'un rapport de gestion faisant office de reddition de compte. Si, généralement, les acteurs universitaires s'entendent sur la nécessité de rendre des comptes, on observe toutefois certaines réticences (Vose, 2014) à appliquer de façon intégrale des mesures perçues comme limitant leur pouvoir de décision et remettant en question les valeurs fondamentales de l'institution (l'autonomie, la liberté universitaire, la collégialité et le choix des contenus d'enseignement et des orientations de la recherche).

Le Rapport du Chantier québécois sur une loi-cadre des universités (Ministère de l'Enseignement supérieur, de la Recherche, de la Science et de la Technologie [MESRST], 2013) fait état de la nécessité de revoir les processus de planification, d'évaluation et de reddition de compte. Ce rapport rappelle l'absence de consensus sur, notamment, les objectifs à atteindre dans le cadre de la planification et sur les indicateurs à utiliser pour la reddition de comptes. La conception de l'université ne peut se réduire à la conception mécaniste d'une organisation hiérarchique et bureaucratique stricte, aux processus de production répétitifs et à une planification rigide des tâches (Fallon et Leclerc, 2014; Fisher, Rubenson, Shanahan et Trottier, 2014). Au-delà de leur triple mission fondamentale (enseignement, recherche et service à la collectivité), le bon fonctionnement des établissements universitaires repose sur l'agencement complexe des valeurs, des objectifs et des actions parfois antagoniques d'une multitude d'acteurs, tant internes qu'externes, le tout couvrant un large spectre disciplinaire (Héon, 1991). Comme l'indiquent Gross et Grambsch (1974) : " the problem is not that institutions cannot identify their goals but rather that they simultaneously embrace a large number of conflicting goals. » Pour rendre compte de la complexité des universités, il faut auparavant clarifier les objectifs antagoniques et spécifier la stratégie pour les atteindre à la lumière des pratiques de gestion et des modèles d'évaluation retenus, c'est à ce problème auquel s'est attelée Larouche (2011) par l'entremise d'une approche typologique.

\section{Description sommaire des conceptions fondant la typologie}

L'essentiel de cette typologie ${ }^{3}$ est présenté dans le tableau 1. 


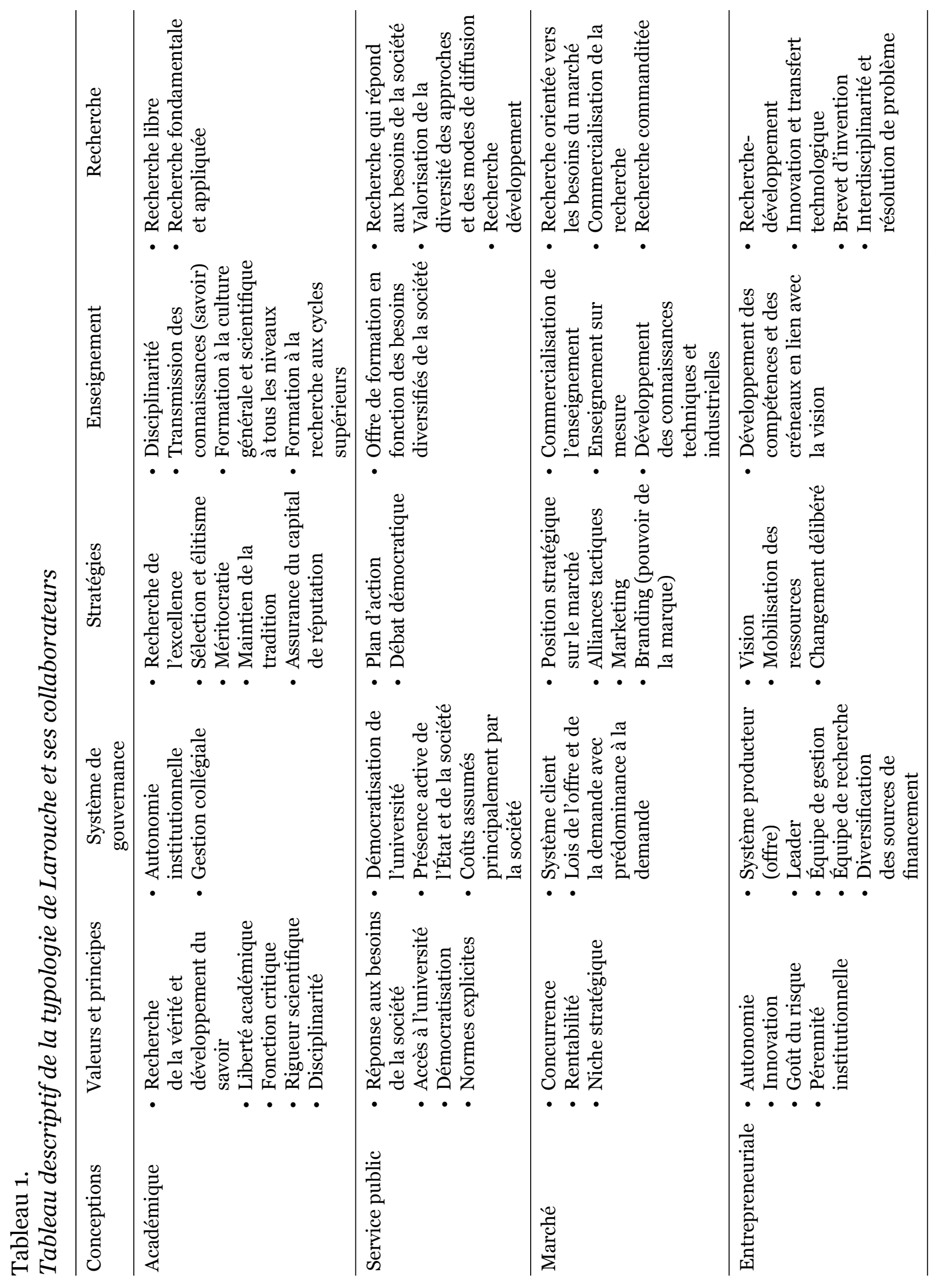




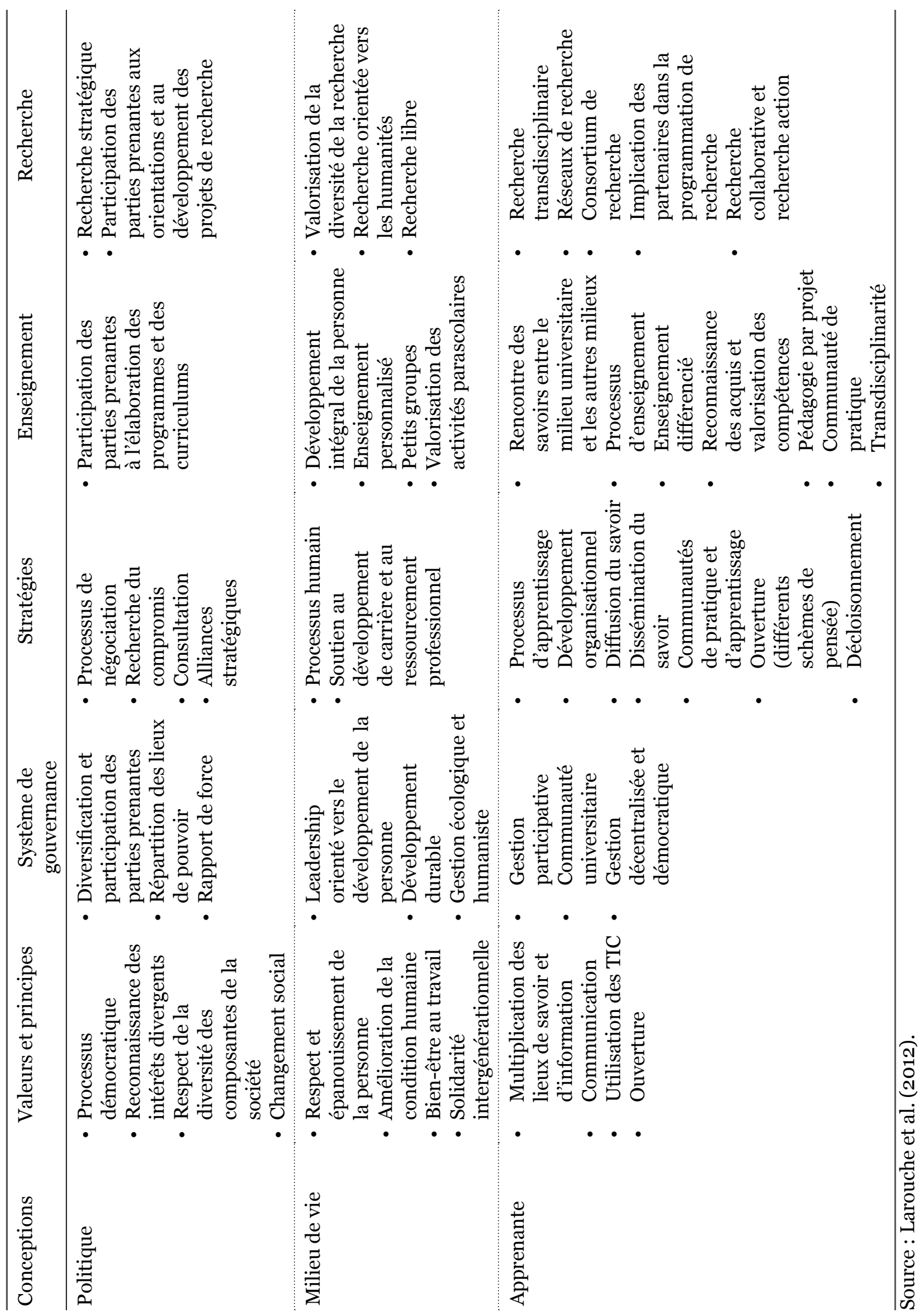


Dans la conception académique, l'université porte et exprime certaines finalités idéales qui transcendent la temporalité et l'espace (Freitag, 2002). Elle concrétise une idée civilisatrice qui vise la formation des citoyens à un idéal d'humanité, de culture et de vie communes (Freitag, 2002). On y recherche la vérité, on valorise le développement du savoir et la rigueur scientifique. Au sein de cette conception, la liberté académique s'avère une valeur fondamentale (Larouche et al., 2012).

Pour la conception service public, l'éducation, l'enseignement supérieur et la formation universitaire constituent un bien personnel et social (Ministère de l'Éducation du Québec [MEQ], 2000). On conçoit l'université au service de tous, comme un bien commun faisant partie du patrimoine collectif (Fédération québécoise des professeures et des professeurs d'université [FQPPU], 1997). L'université vise la réussite et la qualification de ses bénéficiaires. Elle est ouverte sur la société : elle se montre capable de répondre à ses attentes et de contribuer au progrès de la collectivité dans son ensemble (Larouche et al., 2012).

La conception marché décrit une université ayant une valeur marchande (Porter, 1993), soumise aux lois d'un marché autorégulateur. On retrouve la notion de logique de marché dans l'idée de capacité à développer l'innovation afin de répondre à une demande déterminée dans un contexte de concurrence régionale, nationale et internationale. La notion de marché est articulée principalement aux questions de positionnement concurrentiel et de satisfaction de la clientèle, tant sur le plan de l'enseignement que de la recherche et des services à la collectivité (Larouche et al., 2012).

La conception entrepreneuriale vise à exploiter les ressources et les possibilités de développement au-delà des moyens disponibles (Clark, 1998). L'établissement s'adapte à un univers complexe et incertain, mais le fait à ses propres conditions. Cette conception se distingue de la conception marché dans la mesure où elle repose sur la logique de l'offre, marquée par le goût du risque, la créativité, le développement de productions novatrices, alors que la conception marché repose sur la demande et se traduit par des réalisations adaptées aux besoins relevés (Larouche et al., 2012).

Les tenants de la conception politique recherchent la légitimité auprès des parties prenantes (Morin, Savoie et Beaudin, 1994; Quinn et Rorhbaugh, 1983). Il s'agit d'obtenir le soutien et la confiance des acteurs et groupes d'intérêt de son environnement. Les tenants de la conception politique mettent l'accent sur le changement social en luttant contre les forces dominantes et en cherchant l'équité entre les différentes composantes de la société (Larouche et al., 2012).

La conception milieu de vie prône que la pleine réalisation des capacités de l'être humain transite par la connaissance de soi, la liberté d'agir sur sa propre destinée, la légitimité de la recherche du bonheur, la tolérance envers les idées des autres et un idéal commun de justice. Cette interprétation s'appuie sur un partage des traditions, des valeurs et des habitudes propres à la vie de l'organisation (Mintzberg, Ahlstrand et Lampel, 1999). Cette conception suppose un fort sentiment d'appartenance (Larouche et al., 2012).

La conception apprenante s'inspire du concept d'organisation apprenante (Duke, 1992; James, 2000; Mead, 1995; Ramsden et Martin, 1996). L'organisation apprenante crée, acquiert, transfère des connaissances et modifie sa conduite pour refléter des connaissances et des perspectives nouvelles (Dill, 2003; Garvin, 1993; Larouche et al., 2012).

La coexistence de conceptions différenciées traversant les énoncés de la mission fondamentale des établissements universitaires a été reconnue par la FQPPU (2012). C'est aussi l'avis du recteur de l'Université du Québec à Montréal (UQAM), qui reconnaît à la 
typologie de Larouche et ses collaborateurs la capacité d'appréhender la complexité des établissements universitaires (Proulx, 2013). Ainsi, au-delà des missions fondamentales communes aux universités, il est possible de rendre compte de la diversité de modèles et de conceptions des universités dans nos sociétés.

Dans la présente étude, nous émettons l'hypothèse générale qu'au-delà de son utilisation première (l'évaluation de la performance), la typologie peut servir lors des processus de planification stratégique.

\section{Cadre conceptuel}

Nous définissons dans cette section les concepts de mission, de vision et de valeurs dans le cadre plus général des processus de gestion axée sur les résultats et de planification stratégique.

\section{La gestion axée sur les résultats et la planification stratégique}

Le Conseil du trésor (2014) définit la gestion axée sur les résultats comme une approche de gestion qui prend en considération les attentes exprimées par les citoyens en fonction des ressources disponibles et qui vise l'atteinte de résultats en fonction d'objectifs préalablement établis. La planification stratégique, qui nécessite un recul par rapport aux opérations quotidiennes, s'avère l'outil de gouvernance préconisé par les ministères et les organismes pour communiquer leur vision, déterminer leurs orientations, établir leurs priorités, procéder aux choix stratégiques de développement et atteindre des résultats préétablis.

Un plan stratégique s'articule autour de cinq éléments (Conseil du trésor, 2014) :

- une description de la mission et de la vision du ministère ou de l'organisme,

- le contexte dans lequel évolue l'organisme ainsi que les principaux enjeux auxquels il fait face,

- les orientations stratégiques, les axes d'intervention et les objectifs retenus,

- les résultats visés (cibles) au terme de la période couverte par le plan,

- les indicateurs de performance utilisés pour mesurer l'atteinte des résultats.

La gestion stratégique de l'organisation selon Desbiens (2015) est présentée de façon schématique dans la figure 1 .

\section{LA GESTION STRATÉGIQUE DE L'ORGANISATION}

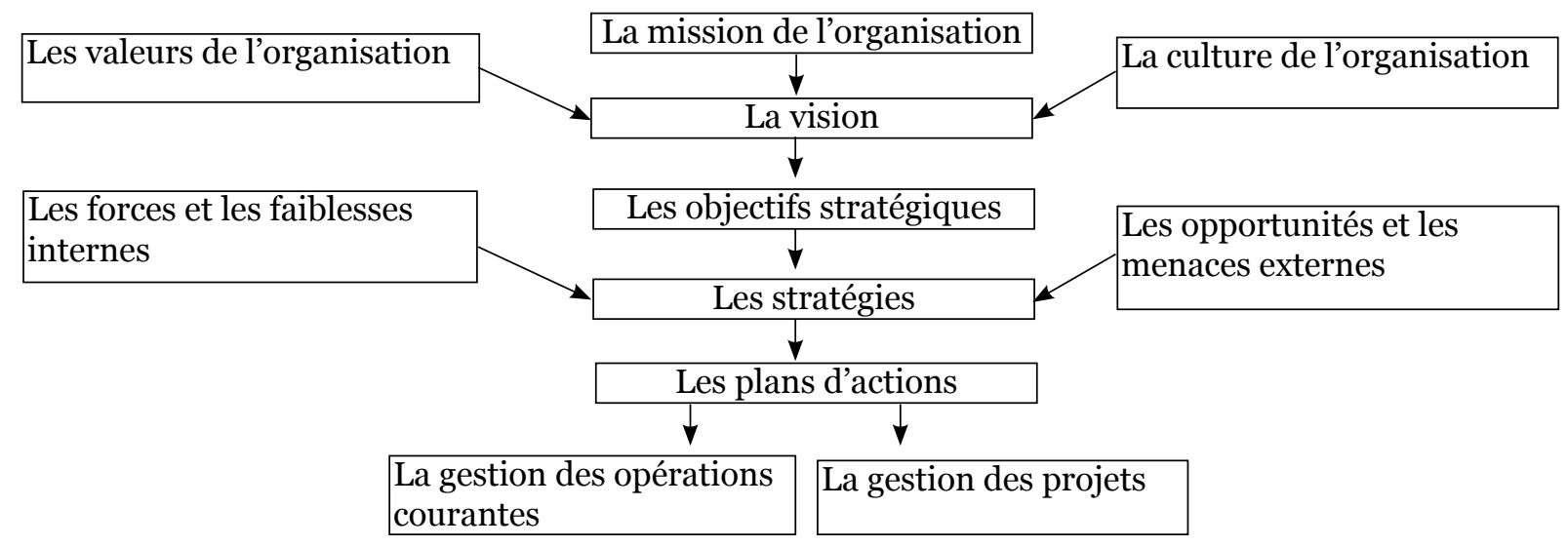

Figure 1. La gestion stratégique de l'organisation selon Desbiens (2015) 
Le processus de planification stratégique prend tout son sens lorsqu'il s'inscrit à l'intérieur de la mission (fondamentale et actualisée) de l'institution universitaire et respecte les principes retenus pour guider son action. On parle d'alignement stratégique lorsque la mission, les valeurs, la vision, les objectifs, les stratégies, les plans d'action et les opérations sont cohérents entre eux. Nous discutons dans la suite du texte des éléments de planification stratégique en contexte québécois.

\section{La mission fondamentale}

$\mathrm{Si}$, au fil du temps et des contextes, les universités ont adopté différentes configurations organisationnelles, elles se sont toujours préoccupées, d'abord et avant tout, de la conservation, de la création, de la transmission et de la diffusion du savoir. Il s'agit du socle commun sur lequel elles ont bâti leur spécificité.

La mission universitaire québécoise repose sur une quadruple fonction sociale (Conseil supérieur de l'éducation [CSE], 2002) ${ }^{4}$ :

1. la formation supérieure des Québécois et des Québécoises (scolarisation et qualification),

2. la création, la conservation, la production, la transmission des savoirs contribuant à l'avancement de l'humanité,

3. l'exercice d'une fonction critique dans la société (développement du sens critique, engagement dans l'action, développement d’une compétence éthique, réflexion épistémologique),

4. l'exercice de rôles connexes (conservation du patrimoine culturel et le développement de la communauté, locale, régionale, nationale ou internationale). Le CSE rejoint ainsi un des principes énoncés dans la Politique des universités du ministère de l'Éducation (Gouvernement du Québec, 1999, p. 17) :

L'université joue un rôle fondamental dans la société. Elle conserve et transmet le savoir et la culture, et les renouvelle. Elle exerce une fonction critique importante. Enfin, par ses ressources et son action elle contribue directement au développement des personnes et dela société. [...] Elle met ses ressources au service dela collectivité.

\section{La mission actualisée}

En 2008, le CSE veut savoir si l'énoncé de mission universitaire qu'il avait proposé en 1995 s'avère toujours pertinent, étant donnéles transformations importantes survenues dans l'environnement et les activités des universités (CSE, 2008). Il rappelle l'évolution de cet énoncé de mission qui, si l'on suit les différents avis émis, doit tenir compte : d'une articulation dynamique entre les volets de la recherche et de la formation (CSE, 1998), d'un ensemble de balises proposées pour guider la mise sur pied et la réalisation des partenariats (CSE, 2002), d'un nouveau rôle connexe dévolu aux établissements (le volet innovation technologique et social) (CSE, 2004) et del'internationalisation des universités (développement durable, aide aux pays en développement, circulation mondiale des cerveaux) (CSE, 2005). Au-delà de la complexité de leur mission, les universités québécoises vivent des tensions économiques liées notamment à leur sous-financement, ce qui les oblige à faire des choix stratégiques majeurs (Proulx, 2009).

Il existe au Québec un consensus assez large autour de la mission et du modèle universitaires à privilégier. Le modèle universitaire québécois se fonde sur une forte 
articulation des activités de formation et de recherche. Les divers établissements partagent aussi des principes basés sur le service public, l'autonomie universitaire et la liberté académique.

Cette position commune s'inscrit dans un système hétérogène d'établissements (CSE, 2008) : universités regroupées dans un réseau public et universités à charte privée; universités offrant le premier cycle exclusivement, les cycles supérieurs uniquement ou l'ensemble des trois cycles; écoles professionnelles et établissements offrant un éventail plus ou moins vaste de domaines; universités de grande taille et de taille plus modeste; universités situées en région et universités des grands centres urbains.

Selon les époques et les circonstances, l'université remplira sa mission différemment, dans un contexte et des conditions en évolution et en transformation (Rocher, 1990). À la mission fondamentale des universités se greffent pour chacun des établissements des valeurs spécifiques qui les amènent à définir différemment l'enseignement, la recherche, la gouvernance, la performance ainsi qu'à privilégier des moyens d'évaluation différents.

\section{La vision}

La vision est la représentation d'une situation qu'on souhaite obtenir dans le futur. Elle englobe un ensemble de croyances et un but général à atteindre. On distingue la vision individuelle (celle d'un gestionnaire, d'un entrepreneur, d'un enseignant) de la vision collective. La vision collective donne un sens à la mission, elle exprime les attentes partagées d'une communauté ou d'une organisation et elle guide l'organisation dans la gestion du changement souhaité (Tardif, 2004). Dans la sphère publique gouvernementale, la vision est articulée à deux axes d'intervention : les priorités gouvernementales (celle des élus au pouvoir) et la finalité de chaque organisation publique (Mazouz et Leclerc, 2008). La vision place au cœur du processus de planification des questions telles que : où sommesnous présentement? Où voulons-nous aller? Où allons-nous? Comment atteindre ce que nous souhaitons?

La vision renforce le processus de planification stratégique en guidant l'élaboration des orientations, des objectifs et des moyens mis en place pour atteindre les buts souhaités, ce qu'illustre la figure 1.

\section{Les valeurs}

Le concept de valeur tire son origine de valere, en latin " être fort ». Selon le Dictionnaire de sociologie (Le Digol, 2007, p. 836) : "plus encore que les normes, les valeurs font aujourd'hui partie du vocabulaire sociologique pour désigner des idéaux ou principes régulateurs des meilleures fins humaines, susceptibles d'avoir la priorité sur toute autre considération ». Pour Boudon et Bourricaud (1982), les valeurs ne sont rien de plus que des préférences collectives qui apparaissent dans un contexte institutionnel et qui, par la manière dont elles se forment, contribuent à la régulation de ce contexte.

Les valeurs sont associées à des préférences qui peuvent varier d'un individu ou d'un groupe à l'autre (" polythéisme des valeurs ») (Le Digol, 2007, p. 836 et 838). Boudon et Bourricaud (1982) considèrent que, si à chaque valeur correspond une orientation propre, la décision est un arbitrage entre ces diverses orientations jamais données "à l'état pur », mais incarnées dans des combinaisons institutionnelles complexes et 
contingentes. Appliqué au monde de l'évaluation, ce « polythéisme des valeurs » implique une évaluation multicritères. Boudon et Bourricaud suggèrent de distinguer, dans tout système de valeurs, le noyau dur des réfractions très variables sous lesquelles ce noyau apparaît dans le temps et en des combinaisons très diverses.

\section{Le cadre d'analyse : les critères de validité d'une typologie}

Nous avons effectué notre analyse de l'applicabilité de la typologie au processus de planification stratégique à partir des six critères de validité d'une typologie de Sauvé (1992).

1. Clarté : les types doivent être clairement définis de façon à pouvoir être repérés sans équivoque par tous les usagers.

2. Consistance logique : les types doivent être de même nature (c'est-à-dire définis à partir des mêmes critères) et être clairement différenciés; le principe éventuel de leur organisation doit être clairement explicité et respecté dans la construction de tous les types.

3. Exhaustivité ou à défaut grand recouvrement : la typologie est exhaustive ou l'ensemble des types doit couvrir le plus d'éléments possible de la population concernée; les critères de classification de la typologie (ses dimensions) doivent tenir compte de tous les aspects descriptifs pertinents de la réalité concernée.

4. Économie : une typologie ne doit contenir que le nombre nécessaire de catégories signifiantes pour classer le plus d'éléments possible, caractéristique assimilable à la parcimonie.

5. Utilité : une typologie doit comporter des catégories signifiantes et, à titre d'outil d'analyse, permettre (entre autres choses) le diagnostic et la prescription et y contribuer.

6. Acceptabilité par les usagers : un processus de validation par des pairs et des experts permet de s'assurer de la composante d'acceptabilité.

\section{Méthodologie}

Nous avons divisé le contenu des plans stratégiques en vigueur de 17 universités québécoises ${ }^{5}$ en fonction des phases de planification stratégique définies par le Conseil du trésor (2014). Nous avons mené notre analyse sur le contenu des sections des plans stratégiques traitant des missions, des visions et des valeurs. Nous avons segmenté ce contenu en unités de sens (mots, phrases, paragraphes) et l'avons codé à l'aide du logiciel QDA Miner, selon un double codage en fonction des conceptions et des dimensions de la typologie. Nous considérons les mentions comme notre unité d'analyse. Une unité pouvant renvoyer à plusieurs mentions de codage, plusieurs codifications ont pu être attribuées à la même unité de sens : le codage des 278 unités de sens relevées dans les plans stratégiques a ainsi donné lieu à 393 mentions. Nous avons compilé et croisé ces mentions par section de texte et par établissement.

Pour répondre aux trois questions de recherche correspondant aux trois critères choisis de validation d'une typologie (clarté et consistance interne, exhaustivité et utilité), nous avons mis en œuvre diverses stratégies.

- Pour la clarté et consistance logique : analyse de concordance inter-juges dans la codification de deux chercheurs. 
- Pour l'exhaustivité : vérification de la fréquence de chacune des conceptions à l'intérieur des mentions codées et détermination des caractéristiques des mentions non classifiables à l'aide de la typologie.

- Pour l'utilité : réalisation de deux analyses sommaires.

La première analyse sommaire liée à la troisième stratégie de recherche a porté sur un aspect diagnostique, soit l'alignement stratégique de l'ensemble des plans des universités par rapport aux conceptions présentes dans les sections relatives à la mission, aux valeurs et à la vision. Dans la seconde, nous avons examiné la capacité de différentiation fournie par la typologie dans l'analyse des conceptions prédominantes relevées dans chacun des plans stratégiques. Les deux analyses sommaires ont été réalisées dans le but d'illustrer le potentiel d'applicabilité de la typologie aux processus de planification stratégique. Nous présentons ici leurs résultats, tant globaux que spécifiques aux établissements, uniquement pour concrétiser ce potentiel, sans aucune prétention quant à leur validité : le lecteur est invité à les consulter avec toute la circonspection requise.

\section{Présentation et discussion des résultats}

\section{Clarté et consistance logique}

La première question de recherche avait comme objectif la vérification de l'univocité de la classification des mentions des plans stratégiques en fonction des conceptions fondant la typologie. Une évaluation de la concordance inter-juges entre deux chercheurs codeurs a montré un degré d'accord de 93,9\%, soit 369 classements identiques sur 393. Cette statistique montre que la typologie présente un niveau de fiabilité élevé, c'est-à-dire que la définition des conceptions est assez claire et logiquement consistante pour que la typologie permette de classer de manière univoque les différentes mentions des plans stratégiques analysés.

\section{Exhaustivité}

La deuxième question de recherche concernait le caractère exhaustif de la typologie et sa capacité à exprimer et à couvrir le réel. Le tableau 2 présente la fréquence et le pourcentage des mentions associées à chacune des conceptions dans les énoncés de mission, de valeurs et de vision des plans stratégiques analysés.

À la lecture du tableau 2, on constate dans les plans stratégiques étudiés la présence, à des degrés divers, de chacune des sept conceptions de la typologie. Cette compilation confirme les résultats antérieurs de Larouche (2011) quant à l'existence, à l'intérieur du système universitaire québécois, de réalités signifiantes reliées à chacune des conceptions. Les conceptions les plus mentionnées ont été, dans l'ordre, la conception académique (25,7 \%) puis les conceptions service public (18,8 \%), milieu de vie (18,8\%), entrepreneuriale $(14,4 \%)$, apprenante $(8,9 \%)$, marché $(4,6 \%)$ et politique $(1,3 \%)$. On retrouve des traces des sept conceptions à la fois dans les énoncés de mission, ceux de vision et dans les valeurs des plans stratégiques étudiés.

Les mentions codées comme appartenant à la conception service public extraites des missions renvoient au développement de la société, au développement régional, aux services à rendre aux collectivités, à l'accessibilité à l'enseignement supérieur par l'offre d'une gamme de programmes étendue à tous les cycles universitaires. Les mentions 
relatives aux idéaux et aux valeurs font référence à une société moderne et démocratique dans laquelle la connaissance est au service du bien commun et du citoyen, dans le respect des différences et de la diversité.

Tableau 2.

Fréquence des mentions relevant de chacune des conceptions au fondement de la typologie de Larouche et ses collaborateurs dans les énoncés de mission, les valeurs et la vision des plans stratégiques des 17 universités québécoises

\begin{tabular}{|c|c|c|c|c|}
\hline $\begin{array}{l}\text { Composantes } \\
\text { Conceptions }\end{array}$ & Mission & Valeurs & Vision & Total \\
\hline Académique & 33 & 46 & 22 & 101 \\
\hline$\%$ pour la conception & $32,7 \%$ & $45,5 \%$ & $21,8 \%$ & $100,0 \%$ \\
\hline$\%$ pour la composante & $22,6 \%$ & $28,8 \%$ & $25,3 \%$ & $25,7 \%$ \\
\hline Service public & 35 & $\mathbf{2 5}$ & 14 & 74 \\
\hline$\%$ pour la conception & $47,3 \%$ & $33,8 \%$ & $18,9 \%$ & $100,0 \%$ \\
\hline$\%$ pour la composante & $24,0 \%$ & $15,6 \%$ & $16,1 \%$ & $18,8 \%$ \\
\hline Milieu de vie & 18 & 39 & $\mathbf{1 7}$ & 74 \\
\hline$\%$ pour la conception & $24,3 \%$ & $52,7 \%$ & $23,0 \%$ & $100,0 \%$ \\
\hline$\%$ pour la composante & $12,3 \%$ & $24,4 \%$ & $19,5 \%$ & $18,8 \%$ \\
\hline Entrepreneuriale & 18 & 23 & 19 & 60 \\
\hline$\%$ pour la conception & $30,0 \%$ & $38,3 \%$ & $31,7 \%$ & $100,0 \%$ \\
\hline$\%$ pour la composante & $12,3 \%$ & $14,4 \%$ & $21,8 \%$ & $15,3 \%$ \\
\hline Apprenante & 12 & 16 & 7 & 35 \\
\hline$\%$ pour la conception & $34,3 \%$ & $45,7 \%$ & $20,0 \%$ & $100,0 \%$ \\
\hline$\%$ pour la composante & $8,2 \%$ & $10,0 \%$ & $8,0 \%$ & $8,9 \%$ \\
\hline Marché & 10 & 3 & 5 & 18 \\
\hline \% pour la conception & $55,6 \%$ & $16,7 \%$ & $27,8 \%$ & $100,0 \%$ \\
\hline$\%$ pour la composante & $6,8 \%$ & $1,9 \%$ & $5,7 \%$ & $4,6 \%$ \\
\hline Politique & $\mathbf{2}$ & $\mathbf{3}$ & $\mathbf{o}$ & 5 \\
\hline$\%$ pour la conception & $40,0 \%$ & $60,0 \%$ & $0,0 \%$ & $100,0 \%$ \\
\hline$\%$ pour la composante & $1,4 \%$ & $1,9 \%$ & $0,0 \%$ & $1,3 \%$ \\
\hline Indéfini & 18 & 5 & 3 & 26 \\
\hline$\%$ pour la conception & $69,2 \%$ & $19,2 \%$ & $11,5 \%$ & $100,0 \%$ \\
\hline \% pour la composante & $12,3 \%$ & $3,1 \%$ & $3,4 \%$ & $6,6 \%$ \\
\hline Total & 146 & 160 & 87 & 393 \\
\hline$\%$ pour la conception & $37,2 \%$ & $40,7 \%$ & $22,1 \%$ & $100,0 \%$ \\
\hline$\%$ pour la composante & $100,0 \%$ & $100,0 \%$ & $100,0 \%$ & $100,0 \%$ \\
\hline
\end{tabular}


Les mentions codées conception académique dans les missions font ressortir la visée de l'excellence en enseignement et en recherche, le rayonnement intellectuel et social de l'institution, l'avancement des connaissances par une démarche rigoureuse et une juste compréhension du monde. Les mentions relevées dans les visions encouragent le développement de la pensée critique. Les mentions ayant trait aux valeurs portent sur l'honnêteté, la transparence, l'autonomie et la liberté académique.

La distinction entre les conceptions marché et entrepreneuriale se dégage nettement de l'analyse. Les mentions attribuables à la conception marché concernent le recrutement des clientèles et un positionnement stratégique misant sur la réponse aux besoins, alors que dans la conception entrepreneuriale, les mentions font référence à la créativité, à l'innovation intellectuelle, scientifique et culturelle dans une optique de progrès, d'augmentation des partenariats et des revenus.

La conception milieu de vie a été décelée à partir de mentions dans les missions qui placent l'étudiant au cœur des préoccupations de l'établissement : son développement global, l'atteinte de son plein potentiel, la réussite de son projet. Cette conception s'inscrit dans une optique humaniste et écologique (de développement durable). Ces mentions décrivent un établissement à échelle humaine, proposent une expérience universitaire engageante et enrichissante qui tienne compte de toutes les facettes de la personne. On relève des valeurs d'éthique, d'équité, d'intégrité, la recherche d'un climat de travail et d'études sain et sécuritaire qui favorise le développement professionnel, le développement du sentiment d'appartenance, la reconnaissance et le respect des personnes, le développement du sens communautaire ainsi que du partage, le tout inscrit dans une gestion harmonieuse et participative.

La conception apprenante transparaît dans les mentions basées sur le concept de communauté apprenante. Ces mentions traitent de l'utilisation et de la promotion des technologies de l'information et des communications, de formation à distance, de décloisonnement des connaissances, de multidisciplinarité, visant l'accentuation des échanges locaux, régionaux et internationaux.

Enfin, nous avons relevé peu de traces de la conception politique, hormis les quelques mentions qui concernent une vision mobilisatrice sensible aux préoccupations des parties prenantes. La nature du document analysé (le plan stratégique) se prête peut-être moins à l'expression d'éléments associés à cette conception que d'autres documents officiels (comme le suivi de la mise en œuvre du plan stratégique).

Par ailleurs, un pourcentage substantiel de mentions n'a pu être associé à un type de conception spécifique (catégorie indéfinie : 12,3\%). On retrouve dans cette catégorie sept énoncés qui traitent de manière générale de la mission fondamentale des universités (formation, recherche et service aux collectivités). Cette mission fondamentale, qui constitue le socle commun des universités, transcende notre typologie, dont la fonction est plutôt de rendre compte des spécificités au-delà de ce socle commun.

On retrouve aussi dans la catégorie des indéfinies cinq mentions relatives aux concepts d'efficience et d'efficacité, insérées par certaines universités au sein de leurs énoncés de mission, de vision et de valeurs. Dans la typologie de Larouche (2011), les notions d'efficience et d'efficacité sont considérées comme des valeurs transversales relevant du domaine administratif. Enfin, cinq mentions non définies relèvent du partenariat, dimension qui demande à être traitée, mais qui n'a pas pu l'être lors de la validation de la typologie. De ce fait, il est pour l'instant impossible d'attribuer ces mentions à une 
conception particulière. Des recherches ultérieures pourraient permettre de spécifier la nature du partenariat en fonction de chacune des conceptions.

\section{Utilité}

Pour la troisième question de recherche, qui portait sur l'utilité de la typologie comme outil d'analyse du processus de planification stratégique, nous avons mentionné que deux analyses sommaires ont été menées, dont nous présentons ici le détail des résultats. La première analyse, globale et en lien avec la qualité du processus de planification, concerne l'alignement stratégique des mentions associées à la mission, à la vision et aux valeurs. La seconde, plus spécifique, porte sur la capacité de la typologie à refléter la diversité des conceptions présentes dans les établissements.

\section{Alignement stratégique.}

La figure 2 rend compte de la répartition des mentions en fonction des sections du plan stratégique.

\section{Ensemble des mentions}

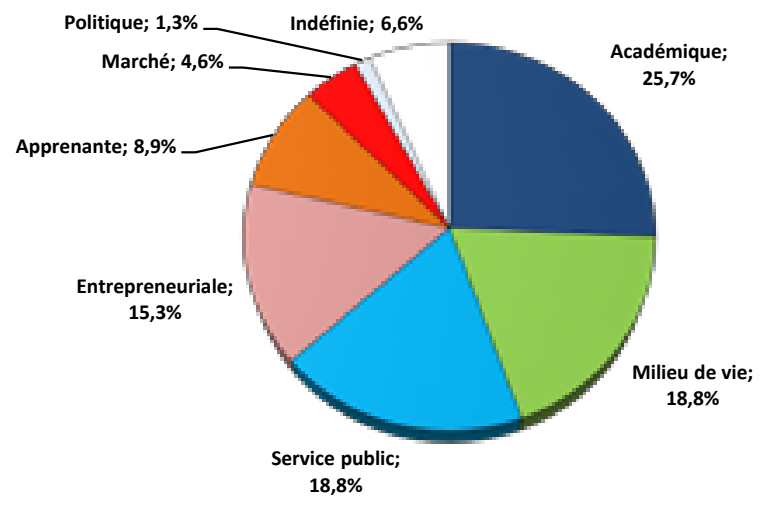

Valeurs

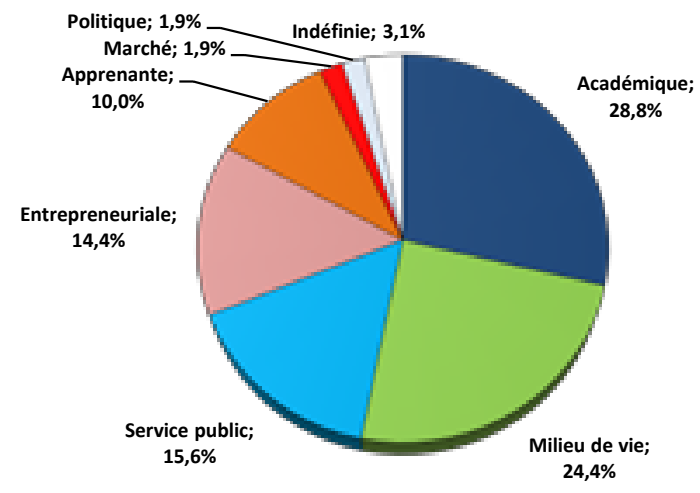

Mission

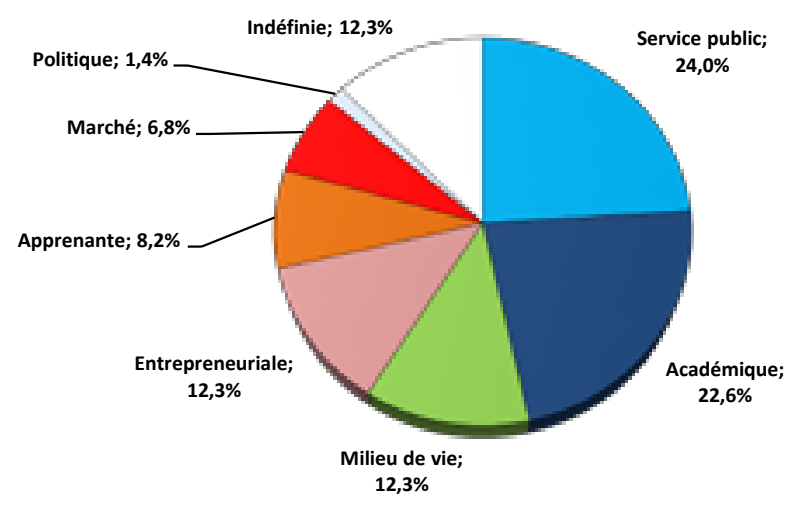

Vision

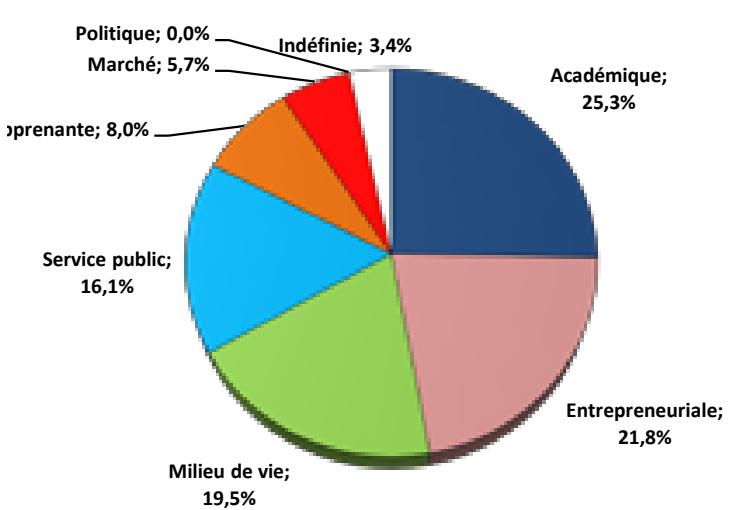

Figure 2. Répartition des mentions en fonction des composantes du plan stratégique (mission, valeurs et vision) 
Dans les sections traitant de la mission, les deux conceptions dominantes sont le service public (avec 24,0 \% des mentions) et la conception académique (mentionnée dans 22,6\% des cas). Sur le plan des valeurs, la conception académique (28,8\%) et la conception milieu de vie $(24,4 \%)$ se sont révélées dominantes. Quant à la vision, les mentions sont partagées entre la conception académique (25,3\%), la conception entrepreneuriale $(21,8 \%)$, la conception milieu de vie $(19,5 \%)$ et la conception service public $(16,1 \%)$, avec un écart moindre entre les conceptions.

Notre analyse révèle également des écarts entre les conceptions relevées dans les énoncés de mission, celles relevées dans les valeurs et celles relevées dans la vision. Par exemple, la conception entrepreneuriale émerge au $2^{\mathrm{e}}$ rang dans les expressions de la vision $(21,8 \%)$ alors qu'elle est moins présente dans l'énoncé de mission $(12,3 \%)$ et dans les valeurs $(14,4 \%)$, résultat qui va dans le sens d'une montée en puissance des pratiques entrepreneuriales dans les universités (Vose, 2014).

Si la vision est construite sur la base des valeurs et qu'elle vise la mise en œuvre de la mission (Desbiens, 2015), les écarts relevés soulèvent des questions quant à l'alignement stratégique des plans étudiés. Ces résultats expriment-ils de possibles contradictions entre les politiques des universités québécoises (dont émanent une mission de service public basée sur des valeurs plus humanistes) et une vision plus entrepreneuriale (mise de l'avant par le gouvernement et les entreprises et reprise dans le discours officiel de certaines universités)? La vision entrepreneuriale vers laquelle ces universités tendent constitue-t-elle une remise en cause des valeurs communes du système universitaire québécois, voire de la mission fondamentale, fragilisant ainsi le consensus convenu autour de la mission et du modèle universitaires à privilégier?

Ces constatations illustrent le potentiel d'utilisation de la typologie comme outil d'analyse du processus de planification, notamment par le biais d'analyses de contenu, d'études de cas ou d'instruments diagnostiques.

\section{Capacité de la typologie à refléter la diversité.}

La figure 3 présente, par université, les pourcentages de mentions relevant des différentes conceptions. Nous avons inclus dans cette figure uniquement les trois conceptions les plus mentionnées pour chaque université, et nous avons retenu les cas d'égalité seulement s'ils étaient supérieurs à $10 \%$.

Cette figure illustre la capacité de la typologie de Larouche et ses collaborateurs à refléter la diversité des conceptions à l'œuvre dans le processus de planification stratégique des universités québécoises. Chacun des types a été relevé comme conception dominante, sauf la conception politique. Quoique diversifiés, les profils d'établissements ont pu être regroupés en quelques types :

- dominante académique (McGill, Université de Montréal, Polytechnique, Université Laval ${ }^{6}$ et Institut national de la recherche scientifique [INRS])

- dominante milieu de vie (Bishop, Université de Sherbrooke et Université du Québec à Chicoutimi [UQAC])

- dominante entrepreneuriale (Concordia et École de technologie supérieure [ETS])

- dominante apprenante (TELUQ)

- dominantes partagées académique et service public (Universités du Québec à Rimouski, en Outaouais et à Trois-Rivières [UQAR, UQO et UQTR]). 
Analyse typologique des plans stratégiques / C. Larouche, D. Savard, L. Héon et J.-J. Moisset 33

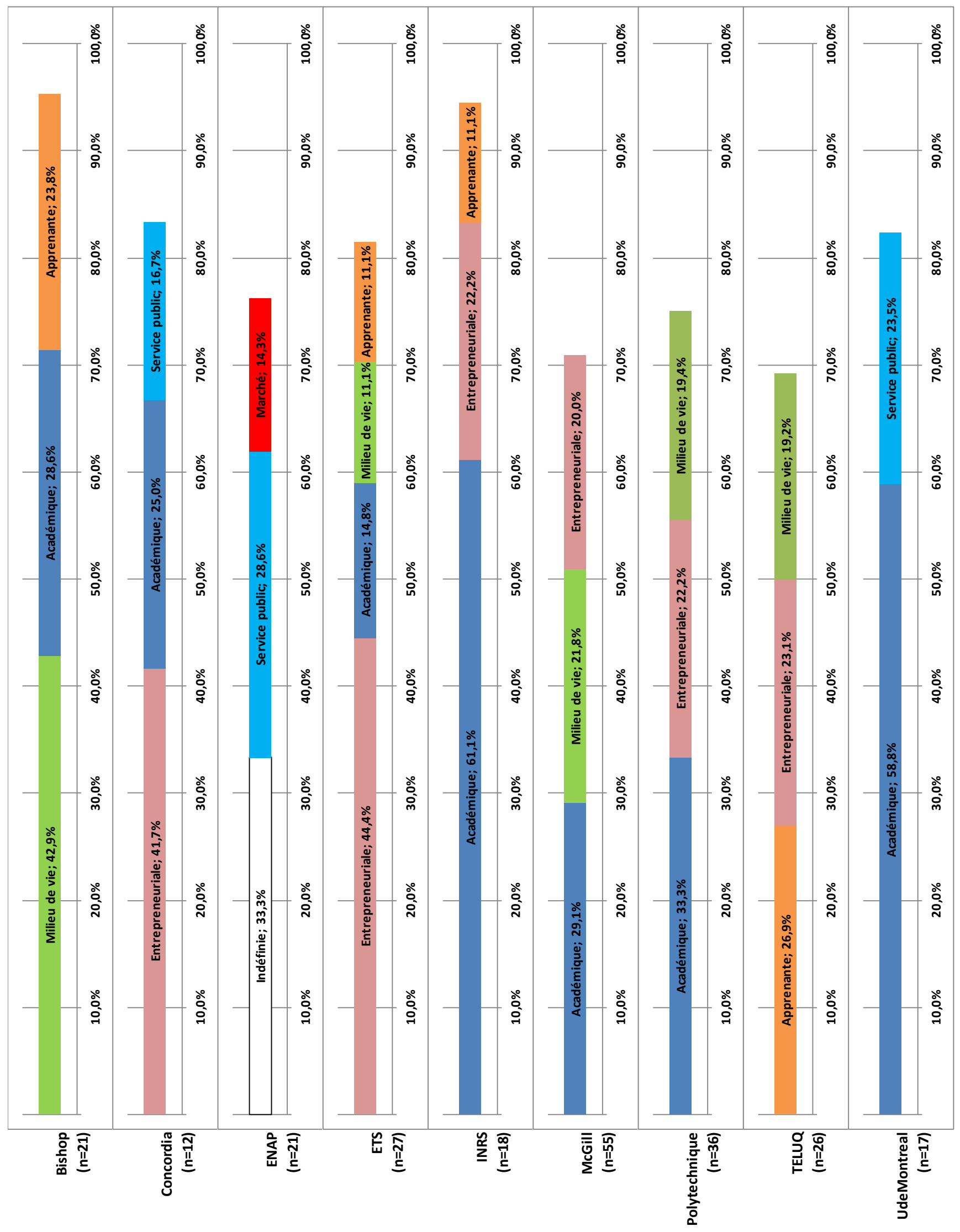




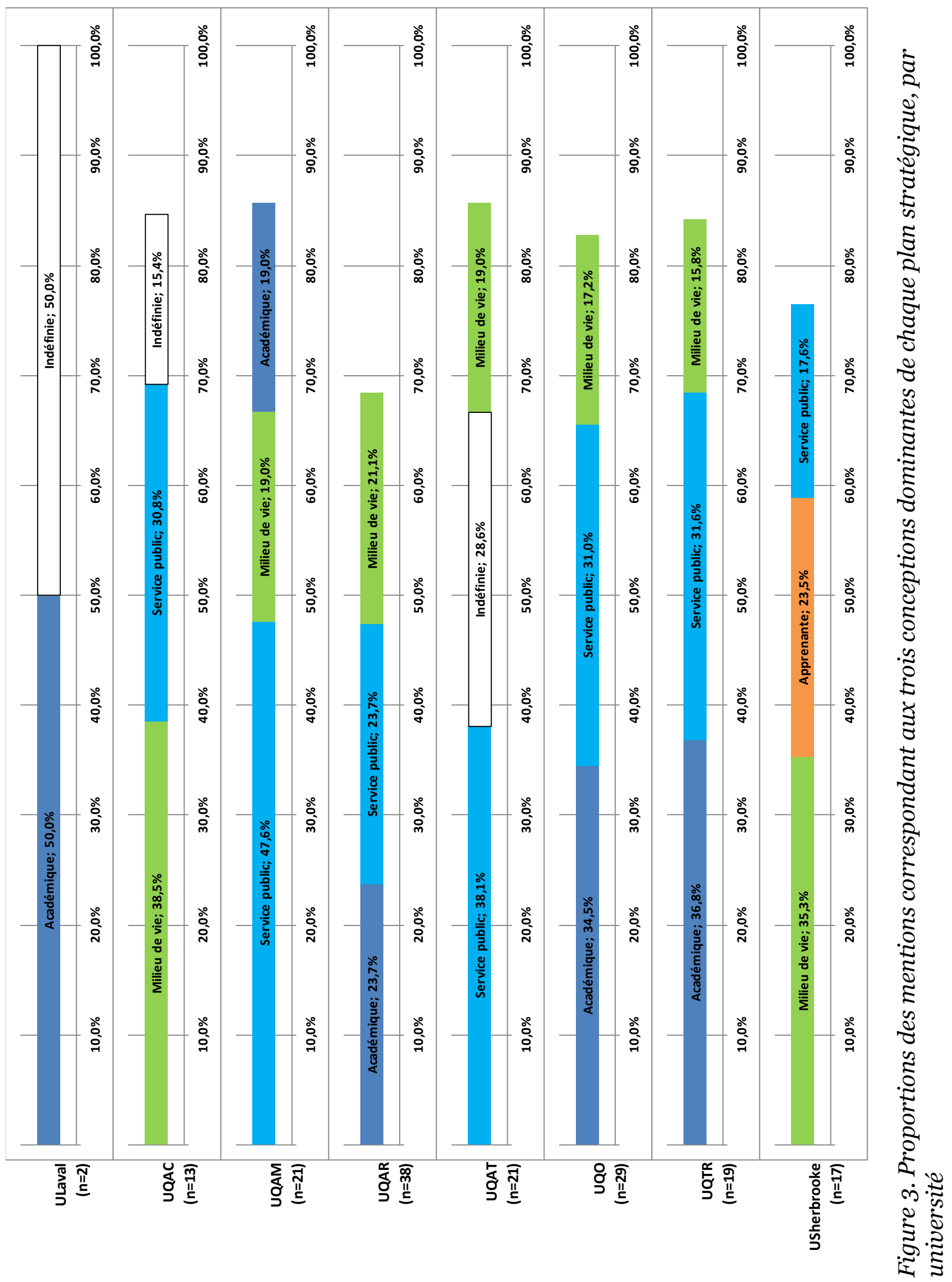


Cette diversité illustre bien le principe de la mission actualisée des universités québécoises, qui reflète la spécificité de chaque établissement. Elle renvoie à la question relevée par le CSE en 2008 sur l'évolution de l'énoncé de mission des universités québécoises adopté par le gouvernement en 2000. Notre étude montre la diversité des valeurs privilégiées par les établissements universitaires québécois telles qu'elles sont mentionnées en planification stratégique. Ces valeurs sont incarnées, comme l'expliquent Bourdon et Bourricaud (1982), dans des combinaisons institutionnelles complexes et contingentes qui illustrent les réfractions très variables de la mission universitaire québécoise. La situation des universités est complexe et diversifiée car elles poursuivent plusieurs objectifs, parfois difficilement conciliables.

Notre exercice d'analyse a permis de mieux comprendre et situer les débats en cours, d'illustrer le sens des négociations entregouvernements, instances de gestion universitaires et membres de la communauté universitaire. À notre avis, l'analyse effectuée aide les dirigeants des établissements visés à clarifier leurs orientations, valeurs et vision pour mieux rendre compte de leur réalité et planifier en conséquence; ce qui est de nature à renforcer l'autonomie institutionnelle.

La prise de conscience d'une tellevariété de contextes et de choix dans les établissements vient renforcer la conviction qu'il est important d'adopter des systèmes d'évaluation de la performance des universités qui soient multidimensionnels et multicritériés. Des recherches supplémentaires dans la validation d'indicateurs de performance plus pertinents et reliés aux conceptions privilégiées par les divers établissements font partie de notre programmation de recherche ${ }^{7}$.

\section{Conclusion}

Cette recherche avait comme objectif de vérifier de manière empirique l'applicabilité de la typologie de Larouche et ses collaborateurs aux exercices de planification stratégique, par le biais d'une analyse de contenu des plans stratégiques des universités québécoises. Nous avons analysé ce corpus à la lumière des critères de validation d'une typologie proposés par Sauvé (1992), à savoir la clarté, la consistance logique, l'exhaustivité et l'utilité, ce qui nous a permis de répondre aux trois groupes de questions ayant alimenté notre réflexion.

1. Les conceptions de la typologie sont-elles assez clairement définies et possèdentelles une consistance logique suffisante pour permettre de classer de façon univoque les différents éléments que l'on retrouve dans les plans stratégiques?

Une évaluation de la concordance inter-juges entre deux chercheurs codeurs a montré un degré d'accord de 93,9\%, soit 369 classements identiques sur 393. La définition des conceptions est assez claire et logiquement consistante pour classer de manière univoque les différentes mentions relevées à l'intérieur des plans stratégiques analysés.

2. La typologie permet-elle de couvrir l'ensemble des éléments présents dans les plans stratégiques? L'ensemble des conceptions de la typologie s'y retrouventelles? Quelles sont les caractéristiques des éléments qui n'ont pas pu être associés à une conception en particulier ou dont le traitement n'a pas été effectué? 
Des mentions des sept conceptions ont été relevées dans les plans stratégiques des universités. Parmi les éléments non définis, on retrouve diverses mentions qui concernent la mission fondamentale des universités comme l'enseignement, la recherche et le service à la collectivité. On repère également les concepts d'efficacité et d'efficience, qui ne relèvent pas de conceptions spécifiques présentes dans la typologie. De plus, le concept de partenariat n'a pu être classé dans aucune conception en particulier, quoiqu'il existe probablement une façon de définir des types de partenariat au sein de chaque conception.

3. La typologie possède-t-elle une sensibilité suffisante pour permettre le diagnostic et détecter la variabilité liée aux différentes situations et pour constituer un outil d'analyse utile?

L'étude a permis de vérifier l'alignement stratégique des énoncés de mission, de vision et de valeurs des divers plans stratégiques. On remarque qu'il y a peut-être un écart entre les conceptions présentes dans les énoncés de mission, les valeurs priorisées par les institutions et les conceptions exprimées dans les énoncés de vision. On peut faire l'hypothèse ici d'une montée en puissance de la conception entrepreneuriale dans les énoncés de vision. Cette vision remet-elle en question le libellé de mission des universités québécoises et les valeurs communes sur lesquelles le socle de cette mission repose? Est-ce le reflet d'un contexte de gestion valorisant ces principes d'action? On pourra ultérieurement mesurer l'alignement stratégique grâce à l'analyse des orientations, objectifs et indicateurs privilégiés par chaque université dans son plan stratégique.

La diversité des conceptions relevées dans les énoncés de mission, de vision et de valeurs des plans stratégiques étudiés est aussi le signe de la persistance du principe d'autonomie des universités par rapport au contrôle gouvernemental et à ses injonctions.

Enfin, ces résultats révèlent la pertinence à adopter des systèmes d'évaluation de la performance des universités qui soient multidimensionnels et multicritériés. Notre programmation de recherche prévoit des études supplémentaires visant la validation d'indicateurs de performance plus pertinents et liés aux conceptions privilégiées par chaque établissement.

\section{Notes}

1. L.R.Q., c.E-14.1.

2. L.R.Q., c. A-6.01.

3. La description des types est tirée de Larouche et al. (2012). On peut en trouver une description plus complète et une discussion détaillée dans Larouche (2011).

4. La définition proposée par le CSE a été légèrement adaptée.

5. Voir en fin d'article la liste des documents consultés.

6. Dans le cas de l'Université Laval, le plan stratégique ne compte que 2 énoncés ayant pu être codés.

7. La programmation de l'équipe de recherche est actuellement subventionnée par le Conseil de recherche en sciences humaines du Canada (CRSH), dossier : 435-20150659; projet:Validation d >une typologie des établissements d enseignement supérieur, 
et spécification d`indicateurs; chercheur principal : Denis Savard; cochercheures : Catherine Larouche et Lucie Héon.

\section{Références}

Boudon, R. et Bourricaud, F. (1982). Dictionnaire critique de la sociologie. Paris : Presses universitaires de France.

Clark, B. R. (1998). Creating entrepreneurial universities: organizational pathways of transformation. Oxford : Pergamon Press.

Conseil du trésor (2014). Le guide sur la gestion axée sur les résultats. Gouvernement du Canada. En ligne à : http://www.tresor.gouv.qc.ca/fileadmin/PDF/publications/ GuideGestionAxeeResultat.pdf

Conseil supérieur de l'éducation (1995). Réactualiser la mission universitaire. SainteFoy : Conseil supérieur de l'éducation.

Conseil supérieur de l'éducation (1998). Recherche, création et formation à l'université : une articulation à promouvoir à tous les cycles. Sainte-Foy : Conseil supérieur de l'éducation.

Conseil supérieur de l'éducation (2002). Les universités à l'heure du partenariat. Sainte-Foy : Conseil supérieur de l'éducation.

Conseil supérieur de l'éducation (2004). Le financement des universités québécoises dans le contexte de l'économie du savoir : un choix de société. Mémoire du Conseil supérieur de l'éducation à la Commission parlementaire sur la qualité, l'accessibilité et le financement des universités. Sainte-Foy : Conseil supérieur de l'éducation.

Conseil supérieur del'éducation (2005). L’internationalisation : nourrir le dynamisme des universités québécoises. Sainte-Foy : Conseil supérieur de l'éducation.

Conseil supérieur de l'éducation (2008). Des acquis à préserver et des défis à relever pour les universités. Avis à la ministre de l'Éducation des loisirs et du sport. Sainte-Foy: Conseil supérieur de l'éducation.

Desbiens, J. (2015). Gestion par résultats. Notes de cours, ENP7332, groupe 11. Québec : École nationale d'administration publique.

Dill, D. (2003). Le paradoxe de la qualité académique: Implications pour les universités et les politiques publiques. Revue des sciences de l'éducation, 29(2), 337-352.

Duke, C. (1992). The learning University: Toward a new paradigm? Buckingham : Society for research into Higher Education et Open University Press.

Fallon, C. et Leclercq, B. (2014) Leurres de la qualité dans l'enseignement supérieur? Variations internationales sur un thème ambigu. Paris : L'Harmattan /Academia.

Fédération québécoise des professeures et des professeurs d'université (FQPPU) (2012). Avis sur la qualité de l'enseignement supérieur en prévision du Sommet sur l'enseignement supérieur. Montréal : FQPPU.

Fisher, D., Rubenson, K., Shanahan, T et Trottier, C. (dir.) (2014). The development of Post secondary education systems in Canada. A comparison between British Colombia, Ontario and Quebec, 1980-2010. Montréal : Mc Gill-Queen's University Press. 
Freitag, M. (2002). L'université aujourd'hui : Les enjeux du maintien de sa mission institutionnelle d'orientation de la société. In G. Gagné (dir.), Main basse sur l'éducation. Montréal : Nota Bene, 294p.

Garvin, D. (1993). Building a learning organization. Harvard Business Revue, 71 (4),78-84

Gouvernement du Québec (1999). Énoncé de politique sur la gestion gouvernementale. Pour de meilleurs services aux citoyens. Un nouveau cadre de gestion pour la fonction publique. Québec : Gouvernement du Québec.

Gross, E. et Grambsch, P. (1974). Changes in University Organization 1964-1971. New York : McGraw-Hill.

Héon, L. (1991). Les forces du marché et de la stratification universitaires sur l'évolution des programmes de premier cycle (le cas des baccalauréats en administration des affaires à l'UQAM et à l'École des H.E.C. (Thèse de doctorat inédite). Université Laval.

James, R. (2000). L'assurance qualité et le difficile problème de la gestion du savoir organisationnel en milieu universitaire. Gestion de l'enseignement supérieur, 12(3), 45-64.

Larouche, C. (2011). La validation d'une typologie des conceptions des universités en vue d'évaluer leur performance. Thèse de doctorat, Université Laval. En ligne à : www. theses.ulaval.ca/2011/27956/27956.pdf

Larouche, C. Savard, D. et Héon, L. (2014). Discours d'experts et typologie des conceptions des universités: la question de la gouvernance. Revue Téléscope, 2O(2), 137-155.

Larouche, C., Savard, D., Héon, L. et Moisset, J.-J. (2012). Typologie des conceptions des universités en vue d'évaluer la performance: Rendre compte de la diversité pour en saisir la complexité. Revue canadienne d'enseignement supérieur, 42(3), 45-64.

Larouche, C., Savard, D., Héon, L. et Moisset, J.-J. (2014). L'anasynthèse comme méthode pour la validation de modèle d'évaluation de la performance appliquée à la validation d'une typologie optimale des conceptions des universités, Revue mesure et évaluation, 37(2), 1-40.

Le Digol, C. (2007). Dictionnaire de sociologie. Paris : Albin Michel.

Mazouz, B. et Leclerc, J. (2008). La gestion intégrée par résultats. Concevoir et gérer autrement la performance dans l'organisation publique. Québec : Presses de l'Université du Québec.

Mead, P. (1995). Utilising the university as a learning organization to facilitate quality improvement. Quality in Higher education, 1(2), 111-121.

Ministère de l'Enseignement supérieur, de la Recherche, de la Science et de la Technologie (MESRST) (2013). Rapport du chantier sur une loi cadre des universités. L'université québécoise : Préserver les fondements, engager des refondations. Québec : Gouvernement du Québec.

Ministère de l'Éducation (2000). Politique québécoise de financement des universités. Québec: Gouvernement du Québec.

Minzberg, H., Ahlstrand, B. et Lampel, J. (1999). Transformer l'entreprise. Gestion, 24(3), 122-131. 
Morin, E. M., Savoie, A. et Beaudin, G. (1994). L'efficacité de l'organisation : théories, représentations et mesures. Montréal : Gaëtan Morin.

Porter, M. E. (1993). L'avantage concurrentiel des nations. Paris : Inter-Éditions.

Proulx, J.-P. (2009). Le système éducatif du Québec. De la maternelle à l'université. Montréal : Chenelière Éducation.

Proulx, R. (2013). Pour en finir avec les conceptions ahistoriques et réductrices de l'université. Introduction à la réflexion sur les finalités des programmes de cycles supérieurs présentée à la commission des études de l'UQAM le 14 mai 2013. Document inédit.

Quinn, R. E. et Rohrbaugh, J. (1983). A spatial model of effectiveness criteria: towards a competing values approach to organizational analysis. Management Science, 29(3), 363.

Ramsden, P. et Martin, E. (1996). Recognition of good university teaching: Policies from an Australian study, Studies in Higher Education, 16(2), 129-150.

Rocher, G. (1990). Re-définition du rôle de l'université. In L'éducation, 25 ans plus tard et après. Actes du colloque tenu à Québec, en novembre 1989, à l'occasion du 25e anniversaire de création du ministère de l'Éducation et du Conseil supérieur de l'Éducation. Québec : Conseil supérieur de l'Éducation.

Sauvé, L. (1992). Éléments d’une théorie du design pédagogique en éducation relative à l'environnement : Élaboration d'un supramodèle pédagogique. (Thèse de doctorat inédite). Université du Québec à Montréal.

Tardif, N. (2004). De l'élaboration de la vision éducative à sa concrétisation. Le Point en administration scolaire, $7(1), 15-19$.

Vose, R.(2014). Differenceisworth fighting for[Internet]. Canada's Vocefor academics, 61(9). En ligne à : https://www.cautbulletin.ca/en_article.asp?ArticleID=3928

\section{Documents consultés}

École nationale d'administration publique (2013-2018). Plan de développement stratégique. Ensemble vers 2018.

École de technologie supérieure (2010-2015). Plan stratégique. ETS au cœur de l'innovation.

Institut national de recherche scientifique (2012-2015). Le plan stratégique. Qualité, innovation et performance.

Polytechnique (2012-2017). Polytechnique Montréal. Le génie en première classe.

TELUQ (2010-2015). Plan stratégique.

Université Bishop's (Document non daté). Mission, vision, valeur. Éducation libérale. Université Concordia (2012-2016). Plan directeur. Enseignement et recherche.

Université de Montréal (2011-2015). Nos grandes orientations. Imaginez ce que sera l'Université de Montréal en 2015.

Université Laval (2013-2017). Orientations de développement de l'Université Laval. Horizon 2017.

Université McGill (2012-2017). Achieving Strategic Academic Priorities. A plan for McGill.

Université Sherbrooke (2010-2015). Plan stratégique Réussir. 
Université du Québec à Chicoutimi (2013-2018). Plan stratégique. Libre de voir plus loin. Université du Québec à Montréal (2009-2014). Plan stratégique.

Université du Québec en Outaouais (2009-2014). Plan stratégique.

Université du Québec à Rimouski (2011-2016). Plan d'orientation stratégique. Soif de synergie.

Université du Québec à Trois-Rivières (2010-2013). Plan stratégique.

Université du Québec en Abitibi Témiscamingue (2009-2014). Plan de développement.

\section{Coordonnées}

Catherine Larouche

Catherine_Larouche@uqac.ca

Université du Québec à Chicoutimi

Catherine Larouche est avocate et professeure agrégée en administration scolaire à l'Université du Québec à Chicoutimi. Elle est titulaire d'un doctorat en administration et évaluation en éducation de l'Université Laval. Elle a reçu en 2012 le prix George-LGeis pour la meilleure thèse de doctorat au Canada dans le domaine de l'enseignement supérieur. Elle est chercheure régulière au Centre de recherche et d'intervention sur la réussite scolaire (CRIRES) et codirectrice de la revue Enseignement et recherche en administration de l'éducation (ERAdE). Elle s'intéresse à l'analyse des pratiques de gouvernance en éducation.

Denis Savard est professeur titulaire en évaluation institutionnelle au Département des fondements et pratiques en éducation de l'Université Laval. Il est chercheur régulier au CRIRES, chercheur associé au Centre de recherche et d'intervention sur l'éducation et la vie au travail (CRIEVAT) et professeur responsable dela Communauté de recherche et d'entraide en éducation (CREÉ), qui compte une centaine de membres en provenance d'une vingtaine de pays. Ses projets de recherche touchent principalement l'évaluation de la performance des établissements d'enseignement et le développement des systèmes éducatifs.

Lucie Héon, détentrice d'un doctorat en administration scolaire, est professeure titulaire au Département des fondements et pratiques en éducation de l'Université Laval. Elle dirige depuis 2010 des programmes d'études supérieures (maîtrises et doctorat) en administration de l'éducation. Elle est chercheure régulière au CRIEVAT (dont elle fut directrice de 2010 à 2012) et coresponsable de la Communauté de recherche et d'entraide en éducation (CREÉ). Ses sujets de recherche sont l'étude des systèmes et des organisations d'enseignement supérieur (planification et gestion) et le travail de gestion.

Jean-Joseph Moisset a obtenu son premier diplôme universitaire en sciences humaines à l'École Normale Supérieure d'Haïti. Il a obtenu depuis un doctorat en économie politique de l'Université de Fribourg (Suisse). Il est professeur émérite de l'Université Laval, où il a poursuivi sa carrière de professeur chercheur (Département des fondements et pratiques en éducation) tout en y exerçant des fonctions administratives et réalisant des actions significatives au niveau international. Membre fondateur de l'Université Quisqueya en Haïti et président de GRAHN-Québec, il est partenaire émérite de la Communauté de recherche et d'entraide en éducation. 